\title{
Nuclear lamina - like filaments and nuclear matrix in Allium cepa as revealed by scanning electron microscopy
}

\author{
HaO Shui, Alin HU, Dezhang Jin, Mingda JiAO and \\ BAIQU HUANG \\ Institute of Genetics \& Cytology, Northeast Normal Universi- \\ ty, Changchun 130024, China
}

\begin{abstract}
In this study, freeze - fractured specimens of Allium cepa root tip meristems were examined under the scanning electron microscope (SEM). This technique permitted the visualization of the outer membrane of the nuclear envelope with nuclear pore complexes and polyribosomes. Some of the cell nuclei prepared with this procedure had fissures of various widths on their nuclear envelopes through which the nuclear lamina- like filaments (LLF) underneath the nucleoplasmic side of the envelopes were clearly visible. The diameters of these filaments varied between 25 and $125 \mathrm{~nm}$. Many of the LLFs showed granular thickenings at places, and were attached to the inner surface of nuclear envelope in some regions. Similar LLFs were also seen at the peripheries of the freeze - fractured faces of nuclei. Meanwhile,the spatial relation between the nuclear matrix filaments (NMF) and other nuclear structures (nucleoli, chromatin and peripheral lamina - like filaments) was revealed in these fractured preparations. In addition, the methods and techniques in studying the nuclear lamina morphology and the roles played by NMFs in activities of various nuclear structures were discussed in brief.
\end{abstract}

Key words: nuclear lamina-like filaments, nuclear matrix filaments, Allium cepa, freeze-fracture.

\section{INTRODUCTION}

It has been evident that in addition to the nuclear envelope, chromatin and nucleolus, a structure composed mainly of protein, termed the nuclear matrix (NM) exists in the nucleus [1,2]. The NM also contains a considerable amount of RNA [3 - 5] and morphologically appears as a filamentous network[6,7]. In a broad sense, the nuclear matrix consists of 3 components, i.e. the nuclear lamina (NL), the internal matrix and 
Lamina like filaments and nuclear matrix in Allium cepa.

the nucleolar residue, although a generally accepted definition of NM has not yet been attained [8]. In a more specific sense, the NL is not included in nuclear matrix [32]. Extensive studies and reviews on the structure and function of NM can be found in the literature[6, 8].However, almost all the studies regarding nuclear matrix used animal or human eells, while the understanding of NM in plant cells is scarce so far. Ghosh and Dey (1986) first observed the NM in A. cepa cells, but in their report only the internal matrix, yet no nuclear lamina was detected[9]. By using immunofluorescence technique, Shaw et al. (1987) were able to provide evidence for the presence of the analogue of lamin in nuclear periphery of A. cepa and Pisum sativum cells,suggesting that a NL structure similar to that found in animal cells may exist in plant cells as well [10].

Recently, Su et al. (1990) first reported the existence of an intermediate filament like framework-lamina-nuclear matrix system in Zea mays protoplasts, though the lamina filament structure wan not clearly illustrated in the photographs of their preparations[11].

In this study, a variety of approaches including the freeze fracture,scanning electron microscopy (SEM) and transmission electron microscopy (TEM) were employed to examine the NM and its relation to the condensed chromatin and the nucleolus in root tip meristematic cells of A, серa, and the 3 dimensional strucutre of LLFs beneath the nuclear envelope was demonstrated.

\section{MATERIALS AND METHODS}

\section{Plant material}

The material used in this study was the root tip meristems of Allium cepa. Segments of root tips, $0.51 .0 \mathrm{~mm}$ long, were excised for both TEM and SEM preparations.

\section{Conventional TEM procedure}

The root tips were fixed in $2.5 \%$ glutaraldehyde in $0.2 \mathrm{M}$ phosphate buffer,pH 7.2 ,for $12 \mathrm{~h}$. Postfixation was carried out by immersing the specimens in $2 \%$ osmium tetroxide in phosphate buffer for $4 \mathrm{~h}$. The specimens were dehydrated through a water ethonal acetone series and embedded in Epon 812. Thin sections were cut and stained with uranyl acetate and lead citrate.

\section{RNP preferential staining}

The glutaraldehyde fixed specimens were dehydrated and embedded conventionally. Thin sections were cut and stained with procedure of Bernhard[11]. Sections were examined under a Hitachi 600 TEM.

\section{Freeze fracture}

The root tip meristems were fixed in $1 \%$ osmium tetroxide in $0.1 M$ phosphate buffer ( $\mathrm{pH} 7.2$ ) for 2 h. Some of the specimens were prefixed in Carnoy's solution (ethanol :acetic acid $=3: 1$ ) for $2.5 \mathrm{~h}$ before osmium fixation. The specimens were washed in 0.1 phosphate buffer, kept in $25 \%$ and $50 \%$ dimethyl sulfoxide (DMSO) solutions each for $30 \mathrm{~min}$. The fracture was performed using a pre cooled knife and hammer on a freeze stage in liquid nitrogen. The fractured specimens were immediately immersed in 50\% DMSO at room temperature, washed thoroughly with double distilled water $\left(\mathrm{dd} \mathrm{H}_{2} \mathrm{O}\right)$ and then treated in $0.1 \% \mathrm{OsO}_{4}$ for $4872 \mathrm{~h}$. The specimens were washed in dd $\mathrm{H}_{2} \mathrm{O}$ and postfixed in $1 \% \mathrm{OsO}_{4}$ for $1 \mathrm{~h}$. After washing in dd $\mathrm{H}_{2} \mathrm{O}$, the specimens were treated in $1 \%$ tannic acid for 30 min to 
Hao Sat el.

enhance the conductivity of the tissue. The specimens were subsequently washed with water and postfixed again in $1 \%$ $\mathrm{OsO}_{4}$ for another hour, dehydrated through an ethanol series and critical - point dried. The specimens were finally plated with gold at a thickness of $20 \mathrm{~nm}$ before they were being examined and photographed on a Hitachi S - 570 SEM.

\section{RESULTS}

\section{The lamina -like filamentous structure beneath the nuclear envelope}

The freeze- fractured tissues prepared with the procedure described above often broke at the interface between the nucleus and cytoplasm resulting in a fracture face as shown in Fig. 1. It can be noticed from this fracture face that many nuclei, and part of the cytoplasm, had been stripped off, leaving cavities with residued of cytoplasm (Fig 1 , arrow A), and this has been referred to as the cell concaves in this report. Meanwhile, in other cells, only the cytoplasm had been removed during the fracture while the nuclei remained at their original places, and this gave rise to the cell convexes as termed

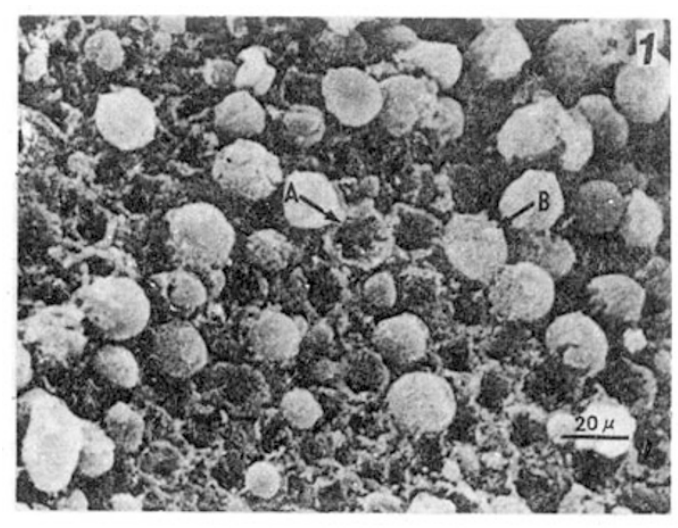

Fig 2. An SEM view of the nuclei in freeze - fractured specimens. In the upper right nucleus, part of its envelope had been removed, so that the chromatin blocks $(\mathbf{C h})$ and nucleolus $(\mathbf{N})$ were exposed. In the lower left nucleus, the nuclear envelope still remained but it was cracked open to form numerous fissures through which the lamina - like filaments underneath the envelope can be seen (arrows L). OM: outer membrane of the envelope.
Fig 1. A low magnification scanning electron micrograph of a freeze - fractured face of a root tip meristem of Allium cepa. Arrow A indicates the cavities resulted from the removal of nuclei during the fracture, while arrow $\boldsymbol{B}$ denotes the convex nucleus after part of the cytoplasm had been stripped off.

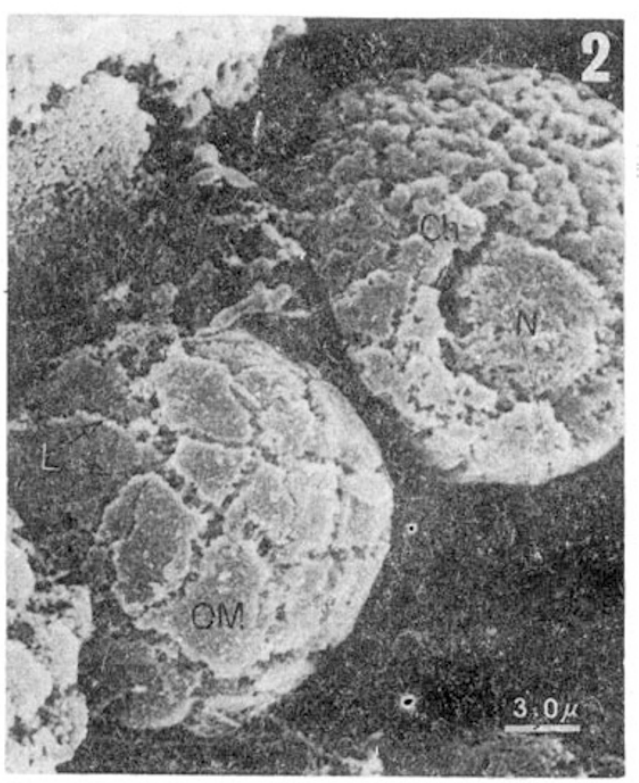


Lamina- like filaments and nuclear matrix in Allium cepa.

in this paper (Fig 1, arrow B). A close examination of the cell convexes under SEM revealed some structural details of the nuclear surface. In some nuclei the nuclear envelopes had been entirely removed and the chromatin $(\mathrm{Ch})$ and nucleolus $(\mathrm{N})$ were exposed (Fig 2), whereas in others the nuclear envelopes cracked open, creating numerous fissures of various widths (Fig 2,--4). Fig 3 and 4 showed the details of the surface of a cell convex. Apart from the nuclear pore complexes (PC), randomly distributed ribosomes $(R)$ or their aggregates can be seen on the outer membrane of the nuclear envelope (Fig 4).
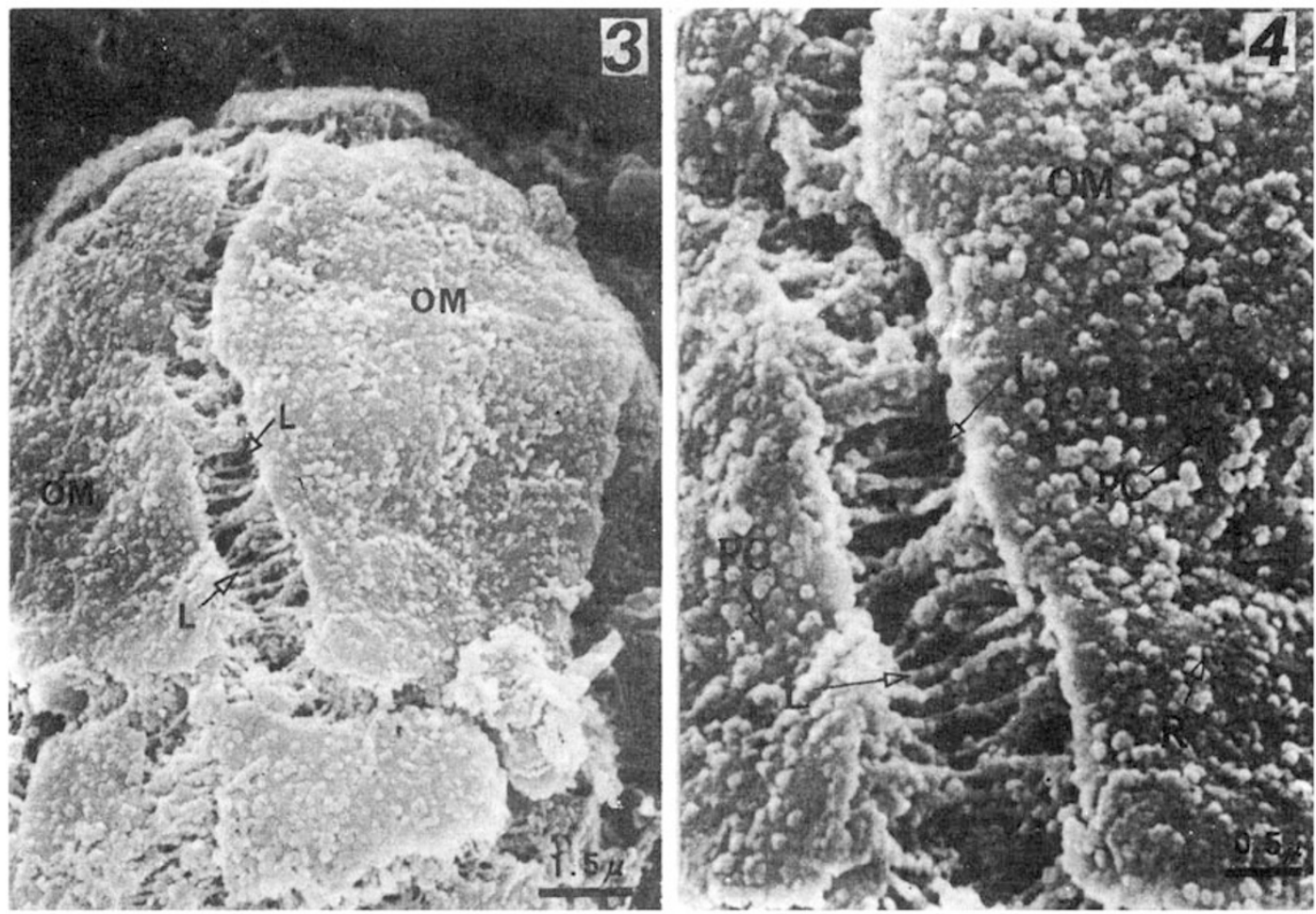

Figs 3 and 4. Freeze-fractured specimens showing the fissures on nuclear envlope and the lamina-like filaments. Fig 4 is an enlarged view of the fissure shown in Fig 3 showing the details of the 3 - D structure of lamina - like filaments.

OM: outer membrane. $\quad$ PC: nuclear pore complexes. $\quad$ L: lamina - like filaments.

R: aggregates of ribosomes.

The majority of nuclei in specimens thus prepared had fissures of various widths on their nuclear envelopes. The fissures were apparently the result of artificial fracture during sample preparation, possibly due to the sudden change in temperature. The presence of these fissures provided the peep - holes into the structures at the nucleoplasmic side of the envelope. The micrographs of Fig 3 - 4 revealed that the lamina - like structure which appeared as a layer of meshwork comprised of filamentous structure underneath the nuclear envelope really existed. Through the SEM stereo pair, it can be clearly seen that this meshwork structure was closely adjacent to the inner side of the envelope (Fig 5), and connected to the envelope in many regions (Fig 4 and 5, arrows). 
Hao S at el.
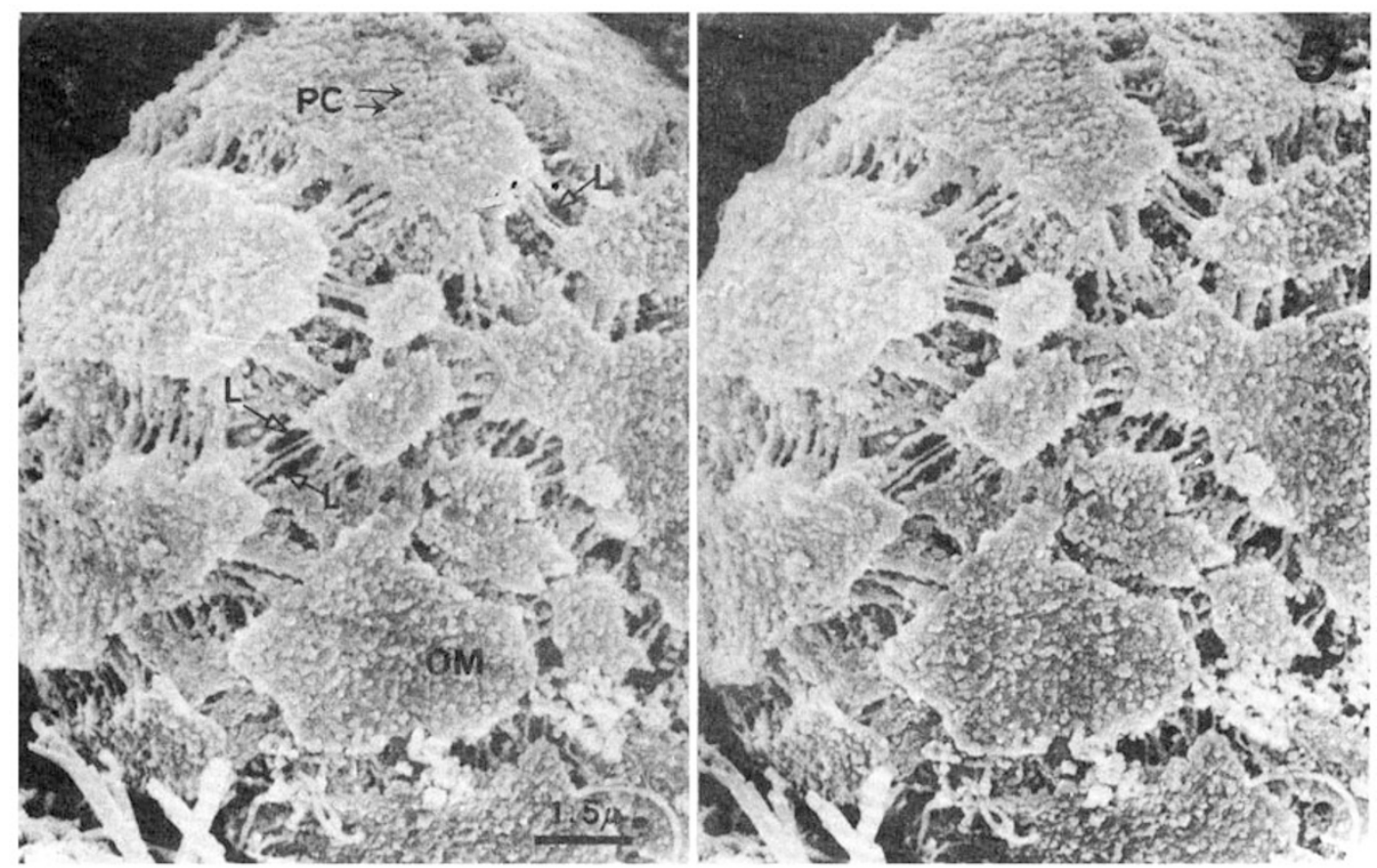

Fig 5. An SEM stereo pair of the onion nucleus in freeze-fractured root tip meristem showing the 3dimensional architecture of the lamina-like structure at the fissures on nuclear envelopes. OM: outer membrane of the envelope. PC: nuclear pore complexes. L: lamina-like filaments.

The thickness of LLFs varied from 25 to $125 \mathrm{~nm}$ with the majority fell between 40 and $80 \mathrm{~nm}$. Many filaments had granular thickenings at places along their length (Fig 4 ). These thickenings were also described for the filaments of NL in animal cells [33].

Besides. the LLFs can be visualized not only through fissures on nuclear surface, but also at the periphery of fracturecl faces of nuclei (see below).

\section{The nuclear matrix filament and its spatial relation to chromatin and nucleolus}

Under TEM, the condensed chromatin and nucleolus can be clearly seen in conventionally stained sections of A. cepa cells, while the nuclear matrix was difficult to identify (Fig 6). The EDTA regressive staining technique of Bernhard, which bleaches the chromatin but contrasts the RNP- containing structure, has been routinely used to demonstrate the nuclear matrix [6,9]. Fig 7 showed a section of $A$. cepa nuclear processed with Bernhard method, in which the nuclear matrix made of fine filaments and granules (arrows) was seen to be distributed between condensed chromatin blocks and the small nucleoli.

The prefixation of specimens in Carnoy's solution before osmium fixation often facilitated the fracture-open of the nucleus during freeze-fracture[4], which enabled the observation of the 3-dimensional organization of the interior structures of nucleus under SEM. Fig 8 represents the fractured face of an interphase cell, showing the LLFs at the periphery of the nucleus (arrows). It can also be seen that the NMFs near 

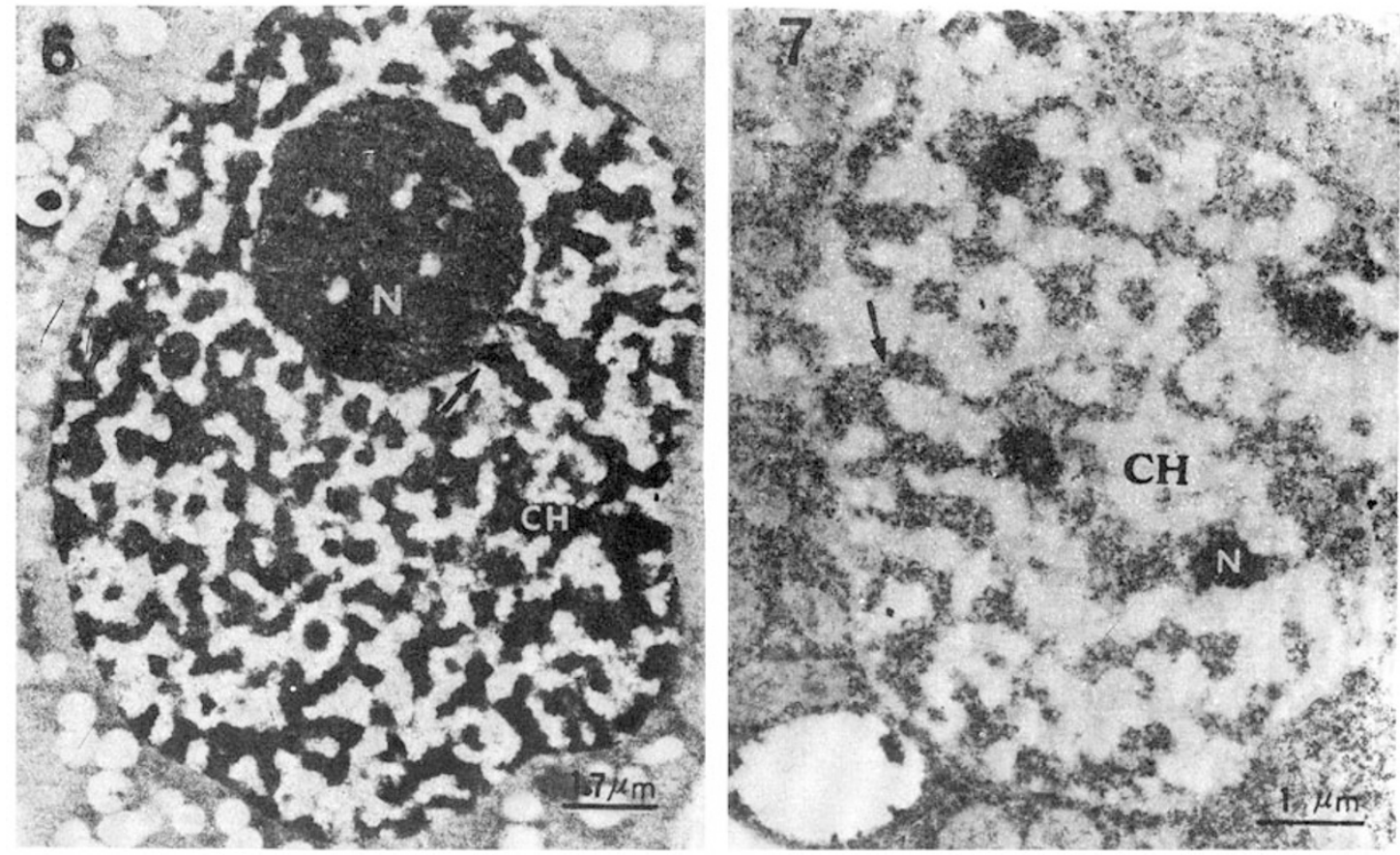

Fig 6. TEM micrograph of the conventionally stained thin section of an interphase cell of root tip meristem of $A$. cepa, showing the chromatin $(\mathbf{C H})$ and nucleoli $(\mathbf{N})$. The nuclear matrix is not visible.

Fig 7. TEM micrograph of an A. cepa root tip meristematic cell which has just entered interphasc. The thin section was stained with Bernhard's method. The bleached chromatin $(\mathbf{C H}), 4$ nucleoli $(\mathbf{N})$ being formed and the nuclear matrix RNP filaments and granules (arrows) are visible.

the nuclear envelope were connected to LLFs at certain angles at one end, and connected to the condensed chromatin blocks at the other (hollow arrows). Since NL is a layer of meshwork structure beneath the nuclear envelope, its filaments should run parallel to the nuclear periphery when viewed at the fractured face. On the other hand, the filaments connected to the LLFs at certain angles and oriented towards the nuclear interior could be taken as the NMFs. Also conspicuous in Figs 8 and 9 were the numerous filaments around the nucleolus, with their other end connecting with the condensed chromatin (arrows). The size of these filaments varied considerably from 20 - $30 \mathrm{~nm}$ (Fig 9) to $50-70 \mathrm{~nm}$. A close examination showed that some of the thicker filaments were composed of 2 or more thinner fibres (Fig 9, arrows). It is apparent that the filaments around the nucleolus were morphologically distinct from the NOR. These filaments were thin and present in large quantity, whereas the NOR usually appeared as blocks in smaller quantity attached to the nucleolus (Fig 6, arrows). Thus, it can be postulated that these filamentous structures represented the nuclear matrix filaments connecting with the nucleoli. Besides, similar NMFs can also be occasionally identified among the condensed chromatin blocks (Fig 8 and 9, arrows). The NMFs observed in Fig 8 and 9 were morphologically distinct from chromatin fibres,since the chromatin fibres in ultrathin sections did not appear as thin, smooth and evenly extended fibres. Moreover, the filaments that we observed in plant cells were very similar to the NMFs 
Hao S at el.

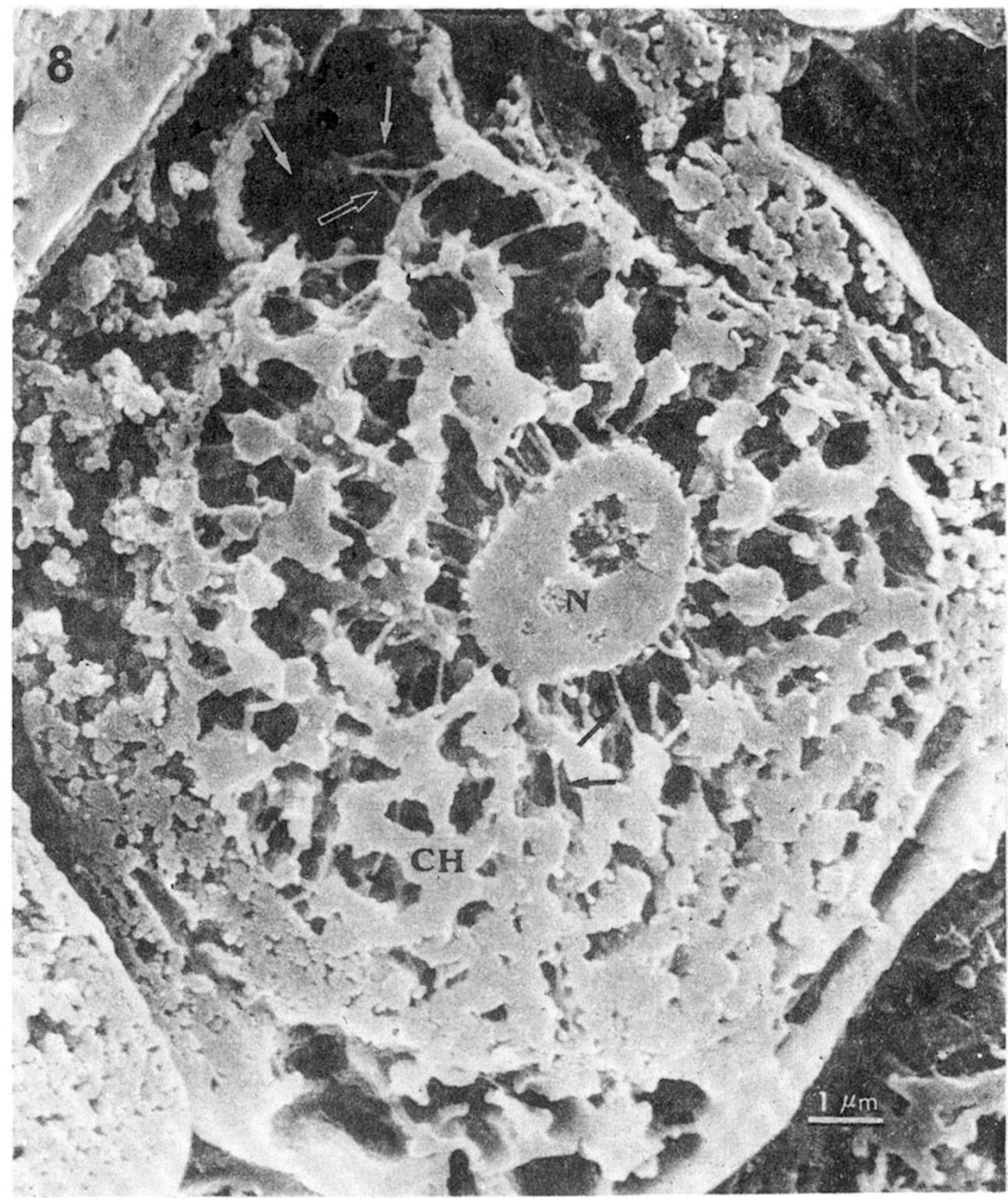

Fig 8. An SEM view of the freeze-fractured face of an onion cell. The arrows pinpoint the lamina-like filaments at the periphery of nucleus. Note the nuclear matrix filaments are connected with lamina-like filaments at certain angles (hollow arrows). Nuclear matrix filaments are also seen to connect between nucleoli $(\mathbf{N})$ and chromatin blocks $(\mathbf{C H})$ (arrows), and among the chromatin blocks as well. 
Lamina- like filaments and nuclear matrix in Allium cepa.

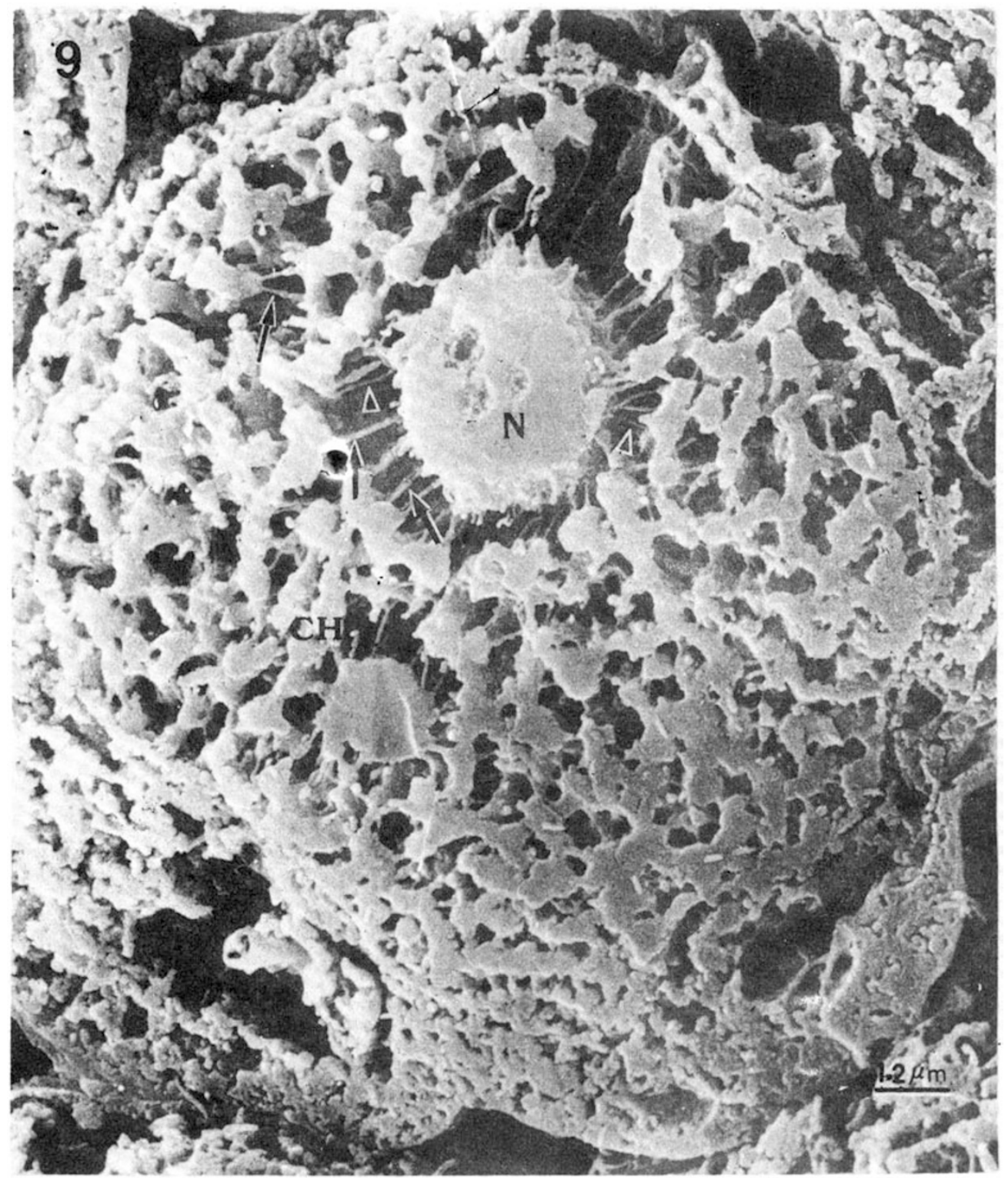

Fig 9. An SEM view of the freeze-fractured face of an onion cell showing the thin nuclear matrix filaments around the nucleolus (arrow head) and between chromatin blocks (CH). Some of the thick filaments are seen to consist of 2 thinner fibres (arrows). 
attached to the chromatin blocks in rat fibroblasts as reported by Penman et al. [32].

In conclusion, in A. cepa root tip cells, the NMFs inter connected the LLFs, the chromatin and the nucleoli within the nucleus.

\section{DISCUSSION}

The nuclear lamina is a layer of filamentous meshwork like structure made up of proteins, and has been found between the chromatin and the nucleoplasmic side of nuclear envelope in the nuclei of a number of animal species [8, 15 20]. The thickness of the lamina has been reported to vary between 30 and $100 \mathrm{~nm}$ [20]. The lamina is composed of 15 special protein species with molecular weights of $60 \quad 70 \mathrm{kD}$, termed lamins, and it has been shown that in laminae in cells of different animals, the constituent lamin species may be different [21 23].

As pointed out by a number of authors, the NL is inter connected with nuclear pore complexes [17,24], the intermediate filaments of cytoskeleton [7,25,26], and the chromatin [27 29]. It has been suggested that apart from its major function in the maintenance of the shape of nucleus and of the special structure of nuclear pore complex, the lamina may also play certain roles in the activities of chromatin and the re construction of nuclear envelope at mitotic telophase [8,21,22].

To study the morphology and location of NL in animal cells, chiefly four approaches have been employed. 1, The thin sectioning and TEM. Although a number of workers had observed the NL by using this procedure, the inherent limitations of thin sections determined that only a very thin fraction of the cell profile was manageable to the observation, and hence it is not an ideal method for the demonstration of nuclear lamina [27]. It has been frequently reported that the NL could not be detected in thin sections $[9,18]$. 2, The immunofluorescence method by the application of antibodies against lamins. This technique allows the demonstration of the existence of a lamin layer adjacent to the nucleoplasmic side of the envelope, but it can not reveal the morphological characteristics of nuclear lamina [30,8]. 3, The use of SEM to examine the nucleoplasmic face of the inner membrane of the broken open nuclear envelope, and a network structure composed of $2040 \mathrm{~nm}$ smooth filaments was observed [16]. This method is able to reveal some 3 dimensional structures of the lamina. 4, Pre treatment of isolated nuclear envelopes with the detergent Triton $\mathrm{x} 100$ for the lysis of certain membrane components, followed by freeze drying and metal shadowing of the specimens, and then examining the remaining lamina and pore complexes under TEM [19]. It has thus been shown that the lamina was a meshwork made of filaments of $10.5 \pm 1.5 \mathrm{~nm}$ in diameter, By using a similar procedure, Stewart and Whytock[13] were able to show that the nuclei of amphibians had essentially the same lamina architecture, i.e. , the meshwork filaments of $10.5 \mathrm{~nm}$ and the beaded thickenings of these filaments.

Attempts at the examination of nucleoplasmic face of the envelope in plant cells have been made only using the thin sectioning and TEM, and no NL was detected [9, 18]. Recently, Shaw et al. (1987) demonstrated the existence of an analogue of lamin at the nuclear peripheries in cells of onion and pea by using immunofluorescence method [10]. In this study, we report, for the first time, the existence of LLFs in plant cells 
Lamina- like filaments and nuclear matrix in Allium cepa.

by means of freeze-fracture and SEM. The thinnest LLFs observed in our preparations were $25 \mathrm{~nm}$ in diameter, very close to the size of lamina filaments in animal cells seen under SEM by Schatten et al. (1976)[31,16], but deviated significantly from the 10.5 nm filaments in animal cells reported by Aebi et al. (1986)[19]. This difference may be the result of different procedures of sample preparation. Our results show that the diameters of the LLFs varied between 25 and $125 \mathrm{~nm}$ and some of the thick filaments were consisted of several thinner fibres (Figs. 4 and 5). We therefore postulate that the thick filaments may be a complex structure.

It should be emphasized that the morphology of NL has been examined only in a limited number of species of animals to date, and the sample preparation in these studies were through diverse procedures. There is no doubt that studies employing a wider variety of materials and applying a broader means of techniques will be more helpful in elucidating the structural and morphological aspects of NL in animal cells. It is noted that there have been no reports so far on the morphology of NL in plant cells, and the LLF structure observed in this study awaits further exploration using other methods.

In an experiment, Capco et al. (1982) used poliovirus to infect cultured human diploid fibroblasts to induce condensation of chromatin, which enabled the visualization of the connection between NMFs and chromatin [7]. Penman (1983) examined the late -prophase $\mathrm{PTK}_{2}$ cells in which the chromatin had been packed into chromosomes allowing the detection of nuclear matrix, and they found that the NMFs were connected with the chromosomes [32]. These authors also managed to digest the HeLa cells with DNase and Observed many NMFs attached to the nucleoli [32].

In this study,we discovered that in interphase nuclei of plant cells, NMFs existed among the condensed chromatin blocks, and between the chromatin blocks and nucleoli as well. Furthermore, our observation showed that the NMFs and LLFs were also inter -connected with each other. Based on these results, and those from experiments on animal cells by other workers, it appeared to us that the nuclear matrix may play certain roles in the maintenance and the coordination of activities of various structures within eukaryotic nuclei.

\section{REFERENCES}

[ 1 ] Aaronson RP,Blobel G. On the attachment of the nuclear pore complex. J Cell Biol 1974; 62:746- 56.

[ 2] Berezney R, Coffey DS. Indentification of a nuclear protein matrix. Biochem Biophys Res Commun 1974; 60: 1410-7.

[ 3 ] Herman R, Weymouth L, Penman S. Heterogeneous nuclear RNA-protein fibers in chromatin-depleted nuclei. J Cell Biol 1978; 78: 663-74.

[4] Berezney R. Fractionation of the nuclear matrix. 1. Partial separation into matrix protein fibrils and a residual ribonucleoprotein fraction. J Cell Biol 1980; 85 : 641-50.

[5] Fey EG, Krochmalnic G, Penman S. The nonchromatin substructures of the nucleus: the ribonucleoprotein (RNP)-containing and RNP-depleted matrices analyzed by sequential fractionation and resinless section electron microscopy. J Cell Biol 1986 ; 102 : 1654-65.

[ 6 ] Berezney R. Organization and functions of the nuclear matrix. In Chromosomal nonhistone proteins-structural associations, Vol. IV (ed L. S. Hnilica) 1984; 119-80. Boca Raton, Florida; CRC Press.

[ 7 ] Capco DG, Wan KM. Penman S. The nuclear matrix: three dimensional architecture and protein composition. Cell 1982; 29:847-58.

[9] Verheijen R, Van Venrooij W, Ramaekers F. The nuclear matrix: structure and composition. J Cell Sci 1988; 90:11-36. 
[ 9 ] Ghosh S, Dey R. Nuclear matrix network in Allium cepa. Chromosoma 1986; 93:429 34.

[10] Shaw P, Rowe A, Rawlins D. Plant nuclear architecture, Annual reports of John Innes Institute, $1987 ; 514$.

[11] Su F, Gu W, Zhai ZH. The keratin intermediate filament like system in maize protoplasts. Cell Res 1990;1:11 6.

[12] Bernhard W. A new staining procedure for electron microscopical cytology. J Ultrastruct Res 1969;27:250 265.

[13] Stewart M,Whytock S. The structure and interaction of components of nuclear envelopes from Xenopus oocyte germinal vesicles observed by heavy metal shadowing. J Cell Sci 1988;90:409 23.

[14] Hu AL, Jing DZ, Jiao MD, Hao S. Effects of different fixatives on freeze fractured samples of plant cells for SEM observation. Acta Bot Sin 1990; 32 (6): 43841.

[15] Dwyer N, Blobel G. A modified procedure for the isolation of a pore complex lamina fraction from rat liver nuclei. J Cell Biol 1976;70:581 91.

[16] Maul GG. The nuclear and cytoplasmic pore complexes : structure, dynamics, distribution and evolution. Intl RevCytol (Suppl) 1977; 6:75 186.

[17] Gerace L, Blum A, Blobel G. Immunocytochemical localization of major polypeptides of the nuclear pore complex lamina fraction. Interphase and mitotic distribution. J Cell Biol 1978; 79:546 66.

[18] Franke WW, Scheer U, Krohne G, Jarasch ED. The nuclear envelope and the architecture of the nuclear periphery. J Cell Biol 1981;91:39s 50s.

[19] Aebi U, Cohn J, Buhle L, Gerace L. The nuclear lamina is a mesh work of intermediate type filaments. Nature $1986 ; 323: 5604$.

[20] Newport JW, Forbes DJ. The nucleus: structure, function and dynamics. Ann Rev Biochem 1987; 56:535 65.

[21] Gerace L, Blobel G. The nuclear envelope lamina is reversibly depolymerized during mitosis. Cell 1980; 19: 27787.

[22] Gerace L, Comeau C, Benson M. organization and modulation of nuclear lamina structure. J Cell Sci (Suppl) $1984 ; 1: 13760$.

[23] Krohne G, Benavente R. The nuclear lamina: a multigene family of proteins in evolution and differentiation. Exp Cell Res 1986; 162:1 10.

[24] Aaronson RP,Blobel G. Isolation of nuclear pore complexes in association with a lamina. Proc Natl Acad Sci USA 1975;72:1007 11 .

[25] Fey EG, Wan KM, Penman S. Epithelial cytoskeletal framework and nuclear matrix intermetiate filament scaffold: three diamensional organization and protein composition. J Cell Biol 1984; 98:1973 84.

[26] Georgatos SD, Blobel G. Lamin B constitutes an intermediate filament attachment site at the nuclear envelope. J Cell Biol 1987; 105:117 25.

[27] Hancock R,Boulikas T. Functional organization in the nucleus,Intl Rev Cytol 1982; 79:165 214.

[28] Bouvier D, Hubert J. Seve AP, Bouteille M. Characterization of lamina born chromatin in the nuclear shell isolated from Hela cells. Exp Cell Res 1985; 156:500 12.

[29] Hubert J, Bourgeois CA. The nuclear skeleton and the spatial arrangement of chromosomes in the interphase nucleus of vertebrate somatic cells. Hum Genet 1986; 74:1 15.

[30] Krohne G,Debus E, Osborn M, Weber K, Franke WW. A monoclonal antibody against nuclear lamina proteins reveals cell type specificity in Xenopus laevis. Exp Cell Res 1984; 150:47 59.

[31] Schatten G, Thoman M, Gerhart J. The nuclear envelope as observed with the scanning electron microscope. J Cell Biol 1976; 70:41a.

[32] Penman S, Capco DG, Fey EG, Chatterjee P, Reiter T, Ermish S, Wan K. The three dimensional structural networks of cytoplasm and nucleus: Function in cells and tissue. In: McIntosh JR ed. Modern cell biology. Alan R Liss Inc New York 1983; 2:385 415.

\section{Received 4-6-1991. Revised 3-1 -1992. Accepted 9-1-1992.}

